\title{
Out, damned dot: studies of the NADPH oxidase in atherosclerosis
}

\begin{abstract}
Kathy K. Griendling and David G. Harrison
Cardiology Division, Department of Medicine, Emory University School of Medicine and the Atlanta Veterans Administration Hospital, Atlanta, Georgia, USA

Address correspondence to: David G. Harrison, Division of Cardiology, Emory University School of Medicine, Room 319 Woodruff Memorial Research Building, 1639 Pierce Drive, Atlanta, Georgia 30322, USA. Phone: (404) 727-3710; Fax: (404) 727-3585; E-mail: dharr02@emory.edu.
\end{abstract}

J. Clin. Invest. 108:1423-1424 (2001). DOI:10.1172/JCI200114453.

There has been growing interest in oxidation reactions in atherogenesis, largely because oxidative modification of lipoproteins is critical for lesion formation. Oxidative events other than lipid modification are likely also important in atherogenesis. Reactive oxygen species (ROS) modulate gene transcription, affect tyrosine phosphorylation, cause DNA damage, and modulate activity of matrix metalloproteinases. The superoxide anion $\left(\mathrm{O}_{2}{ }^{\bullet-}\right)$ reacts rapidly with nitric oxide, resulting in loss of nitric oxide's antiatherogenic properties and formation of peroxynitrite, a strong oxidant. Unpaired electrons (which appear as unpaired dots on Lewis diagrams) are not easily dismissed and often are passed from one molecule to another in radical chain reactions. Many of these redox events occur in either the cell cytoplasm or nucleus and thus may not be affected by interventions targeted to reducing ROS in lipid bilayers or the LDL particle.

The NADPH oxidases are major vascular sources of ROS. These multisubunit enzymes contain a membranebound cytochrome b558-like molecule that is responsible for electron transfer from NADPH to oxygen, producing $\mathrm{O}_{2}{ }^{\bullet-}$. This cytochrome consists of p22phox and the flavoprotein gp91phox or one of its recently identified homologues. Two cytoplasmic subunits, p47phox and p67phox, and the small $G$ protein rac also play regulatory roles. Vascular NADPH oxidase activity is increased in animals with experimental atherosclerosis, and a recent study has shown a strong correlation between NADPH oxidase activity, atherosclerotic risk factors, and endothelial dysfunction (1), suggesting that the NADPH oxidases may be functionally important for atherogenesis.
As they describe in this issue of the JCI, Barry-Lane et al. used NADPH oxidase-deficient mice to investigate a role of these enzymes in atherosclerosis (2). Mice lacking the $p 47^{\text {phox }}$ gene had lower aortic levels of $\mathrm{O}_{2}{ }^{--}$production as compared with wild-type mice. This was confirmed in cultured cells stimulated with serum and thrombin. When p47phox-1- mice were crossed into the apo $E^{-/-}$background, the resulting double knockout mice had significantly less disease in their descending aortas compared with mice lacking only apoE. This difference was evident in mice fed either chow or a high-fat diet. Lack of p47phox had no effect on lesion formation in the ascending aorta, perhaps because lesions develop rapidly in this region and measurements were not made early enough. Nevertheless, these data clearly imply that ROS produced by the NADPH oxidases have a role in lesion development in this model of atherosclerosis.

Two prior studies have examined a role of the NADPH oxidases in atherosclerosis using similar study designs. In one of these, deletion of gp91phox in $a p o E^{-/-}$mice had no effect on the extent of atherosclerotic plaque formation (3). Knockout of $g p 91^{\text {phox }}$, however, may not alter NADPH oxidases in vascular cells, which contain the gp91phox homologues nox 1 and nox 4 that may compensate for loss of gp91phox. In fact, $\mathrm{O}_{2}{ }^{--}$production is not reduced in aortae from $g p 91 p h o x^{-/-}$mice (4). In another study, Hsich et al. (5), like Barry-Lane et al. (2), crossed $p 47^{\text {phox- }-/}$ mice with $a p o E^{-/-}$mice, and they found normal steady-state $\mathrm{O}_{2}{ }^{\bullet-}$ levels in the aorta, normal blood pressure, and equivalent progression of atherosclerosis in the ascending aortae of knockout and wildtype animals on a standard, low-fat diet.
These authors only examined the ascending aorta, a site where atherosclerosis was not different in the study of Barry-Lane (2). Thus, the negative result of Hsich et al. (5) may reflect the site where lesions were examined.

Given the results of Barry-Lane et al. (2) and the basic information showing that oxidative events are important in atherogenesis, one would expect that antioxidant vitamins would either prevent or reduce atherosclerosis in humans. Three recent clinical trials, the GISSI Prevention Trial, the HOPE trial, and the SECURE sub-study of HOPE, however, have shown no effect of vitamin E on cardiovascular outcome and atherosclerosis progression. At face value, these studies seem to cast doubt on the oxidative modification theory of atherosclerosis. There are at least five reasons why we believe that they do not exclude a role of oxidant stress in atherosclerosis, but simply indicate that treatment with vitamin $\mathrm{E}$ is not effective. First, the rate constant for the reaction of vitamin $\mathrm{E}$ with $\mathrm{O}_{2}{ }^{\bullet-}$ is five orders of magnitude slower than the rate of reaction of $\mathrm{O}_{2}{ }^{--}$with endogenous antioxidant enzymes and molecules such as superoxide dismutase and nitric oxide (6). Second, oral intake of vitamin E only modestly increases its plasma and tissue levels (7). Given the slow rate constants for vitamin E's reaction with $\mathrm{O}_{2}{ }^{-}$and other radicals, these modest increases in its concentration are unlikely to affect biological processes. Third, as mentioned above, many of the oxidative reactions that contribute to atherosclerosis occur in the cytoplasm, nucleus, and interstitial space. Vitamin $\mathrm{E}$ is concentrated in lipid bilayers and in the LDL particle and therefore is unlikely to affect these events. Fourth, numerous studies have shown that vitamin E can 
have both antioxidant and pro-oxidant properties, because the tocopheroxyl radical generated when vitamin $E$ reacts with a radical may simply participate in radical chain reactions, rather than stopping them. Finally, treatment with vitamin $E$ in these studies was initiated well after the disease was established, far too late to prevent the events leading to lesion formation.

Considerations such as these and data from experimental animal models, like those presented by Barry-Lane et al. (2), strongly indicate that more research is necessary to definitively test the oxidative stress hypothesis of atherosclerosis in humans. Lipophilic antioxidants such as vitamin E will not eliminate extra unpaired electrons (dots) when they are generated in the cytoplasm or the extracellular space.
The best approach to eliminate these dots is to prevent their production, which would require a better understanding of the enzyme systems involved in their production. It is important to note that angiotensin II and vascular stretch are two of the most potent endogenous stimuli of the vascular NADPH oxidase. Thus, one interpretation of the HOPE trial is that Ramapril, by blocking angiotensin II production and perhaps by lowering blood pressure (and thus preventing vascular stretch), reduced oxidant stress by preventing formation of extra dots latching on to oxygen and sucking dots from other molecules. It is essential that clinical investigators planning future clinical trials understand the complexity of this chemistry of dots.
1. Guzik, T.J., et al. 2000. Vascular superoxide production by NAD $(\mathrm{P}) \mathrm{H}$ oxidase: association with endothelial dysfunction and clinical risk factors. Circ. Res. 86:E85-E90.

2. Barry-Lane, P.A., et al. 2001. p47phox is required for atherosclerotic lesion progression in $\mathrm{ApoE}^{-/-}$ mice. J. Clin. Invest. 108:1513-1522.

3. Kirk, E.A., et al. 2000. Impaired superoxide production due to a deficiency in phagocyte NADPH oxidase fails to inhibit atherosclerosis in mice. Arterioscler. Thromb. Vasc. Biol. 20:1529-1535.

4. Souza, H.P., Laurindo, F.R.M., Ziegelstein, R.C. Berlowitz, C.O., and Zweier, J.L. 2001. Vascular $\mathrm{NAD}(\mathrm{P}) \mathrm{H}$ oxidase is distinct from the phagocytic enzyme and modulates vascular reactivity control. Am. J. Physiol. Heart Circ. Physiol. 280:H658-H667.

5. Hsich, E., et al. 2000. Vascular effects following homozygous disruption of $\mathrm{p} 47$ (phox): an essential component of NADPH oxidase. Circulation. 101:1234-1236.

6. Afanas'ev, I.B. 1989. Superoxide ion: chemistry and biological implications. CRC Press. Boca Raton, Florida, USA. p. 242.

7. Stephens, N.G., et al. 1996. Randomized controlled trial of vitamin $\mathrm{E}$ in patients with coronary disease: Cambridge Heart Antioxidant Study (CHAOS). Lancet. 347:781-786 